\title{
Contaminação por ovos de Toxocara sp. em solo no município de Moreno, Estado de Pernambuco, Brasil
}

José Lancart de LIMA'

Luiz Dias de ANDRADE ${ }^{1}$

Ana Maria AGUIAR-SANTOS'

Leucio Camara ALVES 2

Zulma MEDEIROS ${ }^{1,3}$

Correspondência para:

ZULMAMEDEIROS

Departamento de Parasitologia

Centro de Pesquisas Aggeu Magalhães

Campus da UFPE

Av. Moraes Rêgo s/n - Caixa Postal 7472

50670-420 - Recife - PE

medeiros@cpqam.fiocruz.br

amas@cpqam.fiocruz.br

alves@elogica.com.br

dias@cpqam.fiocruz.br

jlancart@cpqam.fiocruz.br

Recebido para publicação: 06/10/2003 Aprovado para publicação: 23/08/2005

\author{
1 - Departamento de Parasitologia, Centro de Pesquisas Aggeu Magalhães \\ (CPqAM-FIOCRUZ), Recife - PE \\ 2 - Departamento de Medicina Veterinária da Universidade Federal Rural de \\ Pernambuco (UFRPE), Recife - PE \\ 3 - Instituto de Ciências Biológicas da Universidade de Pernambuco, Recife - PE
}

\section{Resumo}

A contaminação do solo por ovos de Toxocara sp. é um importante fator de risco que favorece o surgimento da toxocaríase humana. A infecção se dá através da ingestão destes ovos, embrionados, presentes na água, alimentos ou mãos contaminadas. $\mathrm{O}$ risco da infecção é maior em crianças. O objetivo desse trabalho é determinar a ocorrência de contaminação do solo por ovos de Toxocara sp. na localidade Cidade Evangélica dos Órfãos, pertencente ao município de Moreno-PE, que apresenta casos humanos com sorologia positiva, condições ambientais e sócioeconômicas que favorecem a manutenção do ciclo do Toxocara. Para tal, foram coletadas, por raspagem superficial, 25 amostras de solo, com cerca de $500 \mathrm{~g}$ cada. As amostras foram submetidas à técnica de centrífugo-flutuação em solução de sulfato de zinco a $33 \%$, sendo confeccionadas cinco lâminas por amostra para pesquisa de ovos em microscópio óptico. Das 25 amostras analisadas, 12\% estavam contaminadas com ovos de Toxocara sp. Esses dados demonstram que nesta localidade seus habitantes correm o risco de contrair toxocaríase, requerendo a adoção de medidas que evitem a transmissão entre os animais e entre estes e o homem.

\section{Introdução}

Fezes de animais parasitados, depositados no meio ambiente, podem tornar o solo contaminado com ovos ou larvas de helmintos e oocistos de protozoários, os quais podem provocar doenças em humanos, dentre as quais, podese destacar a Síndrome da Larva Migrans Visceral (SLMV). Beaver et al. ${ }^{1}$ descreveram, em 1952, esta síndrome em três crianças que apresentavam hepatomegalia, febre, manifestações pulmonares e eosinofilia, em analogia à Síndrome da Larva Migrans Cutânea, já bem conhecida na época. Posteriormente, Beaver ${ }^{2}$ definiu a SLMV como sendo o resultado da migração prolongada e persistente de larvas de helmintos em tecidos de hospedeiros não
Palavras-chave

Toxocara canis.

Toxocaríase.

Síndrome da Larva Migrans

Visceral.

Zoonoses. habituais ou paratênicos, especialmente seres humanos, ficando incapazes de completarem o seu ciclo normal e se tornarem adultas, dificultando o diagnóstico da infecção.

Sem dúvida, ascarídeos de cães e gatos, pertencentes ao gênero Toxocara, são os principais responsáveis por causar a SLMV em humanos ${ }^{3}$, por isso a SLMV também é conhecida por toxocaríase, devido aos achados das larvas deste parasito na quase totalidade dos casos diagnosticados por biópsias ou necrópsias em todo o mundo ${ }^{3,4}$. Dentre as espécies do gênero Toxocara, o Toxocara canis, em particular, é reconhecido como o principal agente causador da doença, isso se deve, provavelmente, a diversos fatores, tais como: a grande população mundial de cães; a alta prevalência de Toxocara canis entre esses 
animais; a íntima relação dos cães com o homem, principalmente com as crianças, alvos primários da doença; os hábitos de defecação dos cães; a grande predominância de contaminação do solo com ovos de Toxocara canis, em relação às outras espécies, e o longo tempo de sobrevivência e migração das suas larvas em hospedeiros paratênicos, como o homem ${ }^{3,4}$.

Tomando-se como protótipo o Toxocara canis como o agente etiológico da toxocaríase humana, a dinâmica da transmissão se encontra intimamente associada à relação ecológica: Cão Ambiente - Homem. Assim, aspectos ligados ao homem, ao cão e ao solo, irão constituir os fatores de risco dessa zoonose $\mathrm{e}^{5}$, podendo, então, considerarmos o cão como o principal responsável pela disseminação da toxocaríase. A população canina constitui uma ampla reserva de Toxocara canis, sendo o tamanho desta um dos principais fatores envolvidos na contaminação do solo de uma localidade por ovos de Toxocara canis.

Quando os ovos de Toxocara canis são eliminados, junto com as fezes, estes ainda não se encontram em seu estádio infectante, ou seja, ainda não estão embrionados com larvas de segundo estádio, necessitando, para isto, de condições adequadas de temperatura e umidade. Sob condições ideais, $85 \%$ dos ovos se tornam infectantes após 2 a 6 semanas. A maturação cessa em temperaturas abaixo de $10^{\circ} \mathrm{C}$ e os ovos tornam-se inviáveis a temperaturas de $-15^{\circ} \mathrm{C}^{6,7}$.

Os ovos de Toxocara canis são semiglobosos e possuem uma espessa casca que lhes confere uma longa sobrevida e resistência às adversidades ambientais. Dependendo do tipo de solo e das condições climáticas, os ovos podem permanecer viáveis por longo período ${ }^{8}$. Os ovos de ascarídeos desenvolvem-se melhor em solos de tipo argiloso, principalmente quando não há exposição permanente ou direta à luz solar, que exerce efeito deletério sobre as larvas. No caso particular de ovos de Toxocara também se verifica melhor evolução em solos cuja superfície é constituída por camada argilosa. Os ovos são concentrados pela ação das chuvas, localizando-se em delicada película de lodo, situada logo abaixo da superfície do solo, recobertos por uma fina camada de argila coloidal?

A toxocaríase provoca no homem um quadro clínico bastante variável e inespecífico, resultado dos danos mecânicos ocasionados pelas larvas migrantes e, com freqüência, da resposta imunológica estimulada pela presença da larva e de seus produtos metabólicos. Alguns pacientes apresentamse assintomáticos, enquanto outros apresentam quadro mais grave, caracterizado por febre, hipereosinofilia, hepatomegalia, manifestações oculares, pulmonares ou cardíacas, nefrose e sinais de lesão cerebral ${ }^{6}$. Existem evidências, através de inquéritos sorológicos, de que a infecção por Toxocara canis está bastante disseminada na população humana, sugerindo tratar-se de um problema de saúde pública mundial ${ }^{10}$.

Em estudo soroepidemiológico recente, utilizando-se antígeno recombinante de Toxocara canis, realizado no município de Moreno, Pernambuco, foram analisadas amostras sorológicas de 343 crianças com até 10 anos de idade, provenientes de bairros urbanos e engenhos na zona rural. Neste estudo observou-se que a freqüência de indivíduos com sorologia positiva para anticorpos anti-Toxocara foi de 25,7\%. A maioria dos casos positivos, $14,8 \%$, foi de crianças que residiam na localidade denominada Cidade Evangélica dos Órfãos, área urbana deste município ${ }^{11}$.

Por não ser possível realizar o diagnóstico parasitológico e o diagnóstico sorológico ainda não ser amplamente utilizado pelos serviços de saúde, a toxocaríase humana pode estar subdimensionada e pode representar uma das maiores infecções parasitárias não reconhecidas em nosso meio. Em regiões subdesenvolvidas, como no Nordeste do Brasil, fatores eco-epidemiológicos como: clima quente e úmido, precário saneamento ambiental, baixo nível sócio-econômico, 
elevada população de cães e íntimo contato homem-cão, potencializam o risco de Toxocaríase Humana.

Como a contaminação do solo representa a principal fonte de infecção por Toxocara canis para o homem, este estudo teve como objetivo determinar a ocorrência de ovos de Toxocara sp. no solo da localidade Cidade Evangélica dos Órfãos, situada no município de Moreno, em Pernambuco.

\section{Materiais e Métodos}

A Cidade Evangélica dos Órfãos está situada no distrito de Bonança, pertencente ao município de Moreno, que está localizado na Região Metropolitana do Recife (RMR), Zona da Mata de Pernambuco. O município de Moreno têm uma população de aproximadamente 49.000 habitantes ${ }^{12}$.

A localidade estudada possui uma estrutura com características particulares, onde grupos de crianças e adolescentes, órfãos, residem em um conjunto de casas com precárias condições de higiene e saneamento básico, sob a responsabilidade de um tutor por cada domicílio.

Para a realização deste estudo a localidade foi dividida em cinco quadrantes. Em cada quadrante foram coletadas cinco amostras de solo, em diferentes pontos, totalizando 25 amostras. Cada amostra foi constituída por cerca de $500 \mathrm{~g}$ de terra, coletada por raspagem superficial do solo. As amostras foram coletadas em sacolas de polietileno escuras, devidamente etiquetadas, e transportadas até o local de processamento. $\mathrm{O}$ processamento do material foi realizado no Laboratório de Doenças Parasitárias dos Animais Domésticos da Universidade Federal Rural de Pernambuco (UFRPE) e no Laboratório de Parasitologia do Centro de Pesquisas Aggeu Magalhães, da Fundação Oswaldo Cruz (CPqAM/FIOCRUZ), em Recife - PE.

As amostras de solo foram homogeneizadas individualmente e de cada amostra foram retiradas alíquotas de $50 \mathrm{~g}$.
As amostras foram processadas através do método de Dada ${ }^{13}$, pela técnica de centrífugo-flutuação com sulfato de zinco a $33 \%$. Cada alíquota foi lavada com $50 \mathrm{ml}$ de água, filtrada através de um tamis comum e a suspensão obtida foi dividida em cinco tubos de ensaio de $15 \mathrm{ml}$, que foram centrifugados a 1000 rotações por minuto (r.p.m.) por cinco minutos. Os sobrenadantes foram desprezados e o sedimento de cada tubo foi ressuspendido em solução de sulfato de zinco a $33 \%$, para promover a flutuação dos ovos.

Visando otimizar o processo de flutuação, os materiais foram novamente centrifugados a 1000 r.p.m. por cinco minutos. Ao final da centrifugação, completou-se o volume de cada tubo com solução de sulfato de zinco a $33 \%$, formando um menisco convexo na superfície do líquido, e sobre os tubos foram colocadas lamínulas de vidro deixando-se repousar os tubos por 30 minutos.

Em seguida, as lamínulas foram transferidas para lâminas e observadas ao microscópio óptico, em um aumento de 100x. Foram consideradas positivas as lâminas que continham algum ovo de helminto.

Os resultados obtidos serão entregues, através de relatórios, à secretaria de saúde do município de Moreno e à administração da comunidade Cidade Evangélica dos Órfãos.

\section{Resultados e Discussão}

As amostras de solo utilizadas para análise neste trabalho foram coletadas, estrategicamente, em pontos onde as crianças passam a maior parte do tempo realizando atividades de lazer e educação profissional e de locais nas áreas de peridomicílio (Tabela 1). As 25 amostras de solo coletadas foram, na maioria, do tipo argiloso, com variações de umidade. Nos quadrantes 2 e 3 o solo era argiloso e seco, com presença de pedras, enquanto nos quadrantes 4 e 5 o solo era argiloso e úmido. No quadrante 1 , 
Tabela 1 - Descrição dos quadrantes, características físicas dasárease quantidade de amostras coletadas por quadrante, na Cidade Evangélica dos Órfãos, Moreno-PE, 2003

\begin{tabular}{|c|c|c|}
\hline QUADRANTE & DESCRIÇÃO DA ÁREA & QUANTIDADE DE AMOSTRAS \\
\hline Quadrante 1 & Campo de futebol & 5 \\
\hline Quadrante 2 & $\begin{array}{l}\text { Área de peridomicílio a esquerda do } \\
\text { campo }\end{array}$ & 5 \\
\hline Quadrante 3 & $\begin{array}{c}\text { Área de peridomicílio a direita do } \\
\text { campo }\end{array}$ & 5 \\
\hline Quadrante 4 & $\begin{array}{c}\text { Área de plantio de banana às } \\
\text { margens do lago }\end{array}$ & 5 \\
\hline Quadrante 5 & $\begin{array}{c}\text { Área de plantio de acerola às } \\
\text { margens do lago }\end{array}$ & 5 \\
\hline Total & & 25 \\
\hline
\end{tabular}

Tabela 2 - Descrição dos quadrantesexaminados, características do solo e parasitasencontradosna Cidade Evangélica dosÓrfãos,Moreno-PE,2003

\begin{tabular}{|cccc|}
\hline QUADRANTE & TIPO DE SOLO & N $^{\circ}$ OVOS Toxocara sp & OUTROS HELMINTOS \\
\hline Quadrante 1 & $\begin{array}{c}\text { Arenoso com } \\
\text { pedregulhos } \\
\text { Argiloso e seco, } \\
\text { com presença de } \\
\text { pedras }\end{array}$ & 1 & - \\
Quadrante 2 & $\begin{array}{c}\text { Argiloso e seco, } \\
\text { com presença de } \\
\text { pedras }\end{array}$ & 0 & Ascarididae (5 ovos) \\
Quadrante 3 & Argiloso e úmido & 2 & Ascarididae (3 ovos) \\
\hline Quadrante 5 & Argiloso e úmido & 1 & 8 \\
\hline Total & & 4 & \\
\hline
\end{tabular}

o solo era do tipo arenoso com presença de pedregulhos. Dentre os 5 quadrantes delimitados, 3 se encontravam contaminados por ovos de Toxocara sp., sendo que em dois deles, o tipo de solo predominante foi o argiloso. Estas informações estão descritas no tabela 2.

Dentre as amostras coletadas, três delas foram positivas $(12 \%)$, ou seja, em pelo menos uma lâmina de cada uma das três amostras foi observada a presença de ovos de Toxocara sp. através da técnica de centrífugo-flutuação com solução de sulfato de zinco a $33 \%$.

Além dos ovos de Toxocara sp., também foram encontrados vários ovos da família Ascarididae. Estes ovos foram observados em duas amostras de solo do quadrante 4 e uma do quadrante 5 , ambos com solo do tipo argiloso, também descritos no tabela 2 .

O cão e o gato são animais que conquistaram grande afeto do homem, estando inseridos no meio doméstico, em geral, como suas companhias inseparáveis e fazendo-se presentes nas residências e nos ambientes públicos de lazer. Tendo em vista que as populações canina e felina têm acesso aos locais públicos, livremente ou acompanhados de seus proprietários e, com freqüência, defecam nesses locais, nos chama a atenção para um importante aspecto de saúde pública, tendo em vista o grande potencial de transmissão de doenças que estão envolvidas na relação do homem com esses animais.

Em diversas partes do mundo foram realizados estudos da prevalência de ovos de Toxocara sp. em solo de parques, praias e áreas de uso público e, na maioria das vezes, foram encontrados ovos que revelam a freqüência de contaminação destes locais com ovos do parasita e constitui indicador de risco de infecção $0^{3,10,14,15}$.

O risco de contrair a toxocaríase é maior nas crianças situadas na faixa etária 
entre 2 e 5 anos, que têm contato direto com animais parasitados e com o solo onde foram depositados excrementos destes. Devido aos seus hábitos e suas brincadeiras no ambiente, as crianças podem, facilmente, entrar em contato com ovos de Toxocara canis presentes no solo e ingerirem estes ovos embrionados, presentes em ambientes contaminados, levadas por hábitos geofágicos ou pela freqüência de colocarem em suas bocas seus dedos e outros objetos ${ }^{5}$. A geofagia é um fenômeno observado nas populações rurais e urbanas de vários países e, segundo Pessôa e Martins ${ }^{16}$, constitui-se num hábito arraigado em certas parcelas da população brasileira, traduzindo mais do que puramente uma forma aberrante de ingestão ou perversão do apetite, "um ato de defesa instintiva" do organismo face a deficiência de ferro, cálcio e fósforo, além de outras substâncias minerais. A geofagia é mais freqüente em crianças de áreas pobres, mas, também, se verifica em adultos. Outros fatores, incluindo distúrbios emocionais, retardo mental e disfunções orgânicas cerebrais podem predispor à pica (perversão do apetite observada no decurso de certos estados patológicos) e, consequentemente, a uma maior susceptibilidade à infecção por Toxocara canis ${ }^{8}$.

A determinação da prevalência de contaminação do solo depende do tipo deste solo e da técnica utilizada para recuperação dos ovos, apresentando, por isso, uma ampla variação $0^{3,10,14,15,17}$.

Em 1976, Chieffi e Müller ${ }^{10}$ coletaram amostras de solo de 15 praças da cidade de Londrina-PR e compararam duas técnicas de recuperação de ovos: uma que utiliza sulfato de zinco a $33 \%$ e outra que utiliza dicromato de sódio. Eles observaram a presença de ovos de Toxocara sp. em 60\% das amostras analisadas e concluíram que a técnica com dicromato de sódio foi mais sensível . Em um estudo realizado na cidade de Botucatu-SP, onde foram analisadas 120 amostras de solo coletadas de parques e praças públicas, utilizando a técnica de flutuação em solução decinormal de hidróxido de sódio, observou-se a presença de ovos de Toxocara sp. em 17,5\% das $\operatorname{amostras}^{17}$.

Coelho et al. ${ }^{3}$ coletaram amostras de solo de 30 praças da cidade de SorocabaSP e, utilizando a técnica de flutuação com solução saturada de sulfato de magnésio com 5\% de iodeto de potássio, observaram a presença de ovos de Toxocara sp. em 53,3\% delas.

Oitocentas amostras de solo de praças foram coletadas em várias cidades na Inglaterra e observou-se $24,4 \%$ de positividade, utilizando-se a técnica de recuperação de ovos por flutuação em solução de sulfato de zinco a 33\% \% ${ }^{6}$. Em 1994, Costa-Cruz, Nunes e Buso ${ }^{15}$, analisando 39 amostras de solo de praças, na cidade de Uberlândia-MG, encontraram $23 \%$ de positividade, utilizando a técnica de flutuação em solução saturada de cloreto de sódio (Método de Willis).

Por não existir um protocolo definido e eficiente, para utilizar como técnica de recuperação de ovos de geohelmintos do solo, a comparação dos resultados obtidos nas pesquisas é extremamente difícil. A opção pelo método utilizado em nosso estudo tem como fundamento a publicação de Dada ${ }^{13}$, que comparou vários métodos, inclusive o que utiliza dicromato de sódio, e verificou ser o de sulfato de zinco a $33 \%$ o de maior sensibilidade.

O tipo de solo influencia, sensivelmente, os resultados dos métodos de recuperação de ovos de helmintos no solo ${ }^{18}$. Na Cidade Evangélica dos Órfãos, nos quadrantes que possuíam solo do tipo argiloso, foram observadas as maiores quantidades de ovos recuperados, confirmando os dados encontrados na literatura sobre essa associação?

A presença de ovos de Ascaris sp. no solo da localidade estudada, associada ao alto índice de prevalência das parasitoses intestinais na população de Moreno ${ }^{19,20}$, onde o $A$. lumbricoides foi o helminto mais prevalente, reforça a existência de um mecanismo de contaminação por ovos de 
geohelmintos neste município. Por analogia a este mecanismo, o restante da população de Moreno, não apenas a população da comunidade Cidade Evangélica dos Órfãos, está exposta a um determinado risco de contrair a SLMV.

O encontro de ovos de Toxocara sp. no campo de futebol, área onde a maioria das crianças da Cidade Evangélica dos Órfãos brincam, e nas áreas onde são realizadas práticas agrícolas profissionalizantes, demonstra que os indivíduos positivos encontrados no inquérito soroepidemiológico, realizado por Bezerra ${ }^{11}$, podem ter como local da contaminação o solo destas áreas.

É necessário realizar uma monitoração mais abrangente do problema na área, afim de identificar outros fatores de risco que contribuem para infecção e ou doença no homem por Toxocara cani ${ }^{21}$ bem como avaliar a ocorrência de contaminação do solo em outros locais do município de Moreno e a freqüência de contaminação da população canina local.

O fator determinante da transmissão da toxocaríase seria a presença de ovos embrionados nas amostras de solo positivas. Entretanto, os resultados deste estudo, associados ao estudo de soroprevalência realizado na localidade, reforça a tese de que existe o risco de contrair a toxocaríase na comunidade Cidade Evangélica dos Órfãos.

Ainda são pouco freqüentes os estudos de base populacional com o objetivo de determinar os índices de prevalência da infecção por Toxocara canis e identificar fatores de risco associados a essa parasitose. A prevalência da infecção humana é pouco conhecida, o diagnóstico difícil de ser confirmado, devido aos sinais clínicos serem inespecíficos ${ }^{22}$. O conhecimento da freqüência e do tipo de doença produzida por Toxocara sp. tem aumentado bastante com o tempo. Embora ainda subdiagnosticada e subnotificada, a Síndrome da

\section{Conclusões}

Os resultados deste trabalho demonstram que é necessário fazer o controle e a prevenção da toxocaríase em animais de estimação e no homem, requerendo a adoção de medidas que evitem a transmissão entre os animais e destes para o homem, e que reduzam a contaminação ambiental com ovos de Toxocara sp.

Portanto, faz-se necessário a redução do número de cães e gatos vadios e a prevenção da defecação dos animais em pavimentos, áreas de recreação ou parques infantis, o que se conseguiria colocando grades ou telas ao redor dos parques e com educação sanitária acerca do potencial zoonótico dos parasitas de cães e gatos, além do tratamento destes animais com drogas anti-helmínticas adequadas, sobretudo no caso de filhotes de cães.

O perigo que as infecções por Toxocara canis representam para a saúde pública ainda é pouco conhecido pelos criadores de animais de estimação. $\bigcirc$ médico veterinário é um dos profissionais que devem divulgar as medidas a serem adotadas para diminuir os riscos de contaminação, informando aos clientes e à sociedade sobre o potencial zoonótico dessa parasitose, sobre o ciclo biológico do parasita e a forma de transmissão ao homem, especialmente nas crianças que são as mais freqüentemente acometidas.

\section{Agradecimentos}

Agradecemos à Prefeitura da Cidade de Moreno e à administração da comunidade Cidade Evangélica dos Órfãos, pela cooperação na realização deste trabalho, e a Maria Aparecida da Glória Faustino, professora adjunta da Universidade Federal Rural de Pernambuco, pelas orientações e treinamento parasitológico.

\section{Soil contamination with Toxocara sp. eggs in Moreno, Pernambuco State, Brazil}

\section{Abstract}

The contaminated soil with Toxocara sp. eggs is an important factor
Key-words

Toxocara canis.

Toxocariasis. 
of risk to development of the zoonotic disease. Human infection is acquired by ingestion of embryonated Toxocara sp. eggs with contaminated water, food and also with dirt hands contaminated too. Usually Toxocara sp. infected dogs and cats, but sometimes humans can be affected, mainly children, causing visceral larva migrans. The goal of this study was determine the prevalence of Toxocara eggs in the sand of the locality Cidade Evangelica dos orfaos, Moreno County, northeast of Brazil. Twenty-five soil samples were collected and analyzed by centrifugal flotation technique by using zinc sulfate solution at $33 \%$. The analysis of soil samples showed $12 \%(3 / 25)$ of some contamination level of sand by Toxocara sp eggs. As human can be infect by accidental ingestion of embryonated Toxocara eggs, the results of this research suggest the adoption of measures who prevent the transmission of this zoonotic disease.

\section{Referências}

1 BEAVER, P. C. et al. Chronic eosinophilia due to visceral larva migrans - Report of three cases. Pediatrics, v. 9, p. 7-19, 1952.

2 BEAVER, P. C. The nature of visceral larva migrans. Journal of Parasitology, v. 55, p. 3-12, 1969.

3 COELHO, L. M. P. S. et al. Toxocara spp. eggs in public squares of Sorocaba, São Paulo state, Brazil. Revista do Instituto de Medicina Tropical de São Paulo, v. 43, n. 4, p. 189-191, 2001.

4 ANDRADE, L. D. Aspectos clínicos-epidemiológicos da toxocaríase humana. Revista de Patologia Tropical, v. 29, n. 2, p. 147-159, jul-dez. 2000.

5 AGUIAR-SANTOS, A. M. Toxocaríase humana: soroprevalência em crianças e adolescentes atendidos no Ambulatório do Centro de Pesquisas Aggeu Magalhães - Recife-PE. 2001. 104 f. Dissertação (Mestrado em Pediatria) - Centro de Ciências da Saúde, Universidade Federal de Pernambuco, Pernambuco, 2001.

6 OVERGAAUW, P. A. M. Aspects of Toxocara epidemiology: human toxocarosis. Critical Reviews in Microbiology, v. 23, n. 3, p. 215-231, 1997.

7 SCHANTZ, P. M.; GLICKMAN, L. T. Ascarideos de perros y gatos: un problema de salud publica y de Medicina Veterinaria. Boletin de La Oficina Sanitaria Panamericana, v. 94, p. 571-586, 1983.

8 GLICKMAN, L. T.; SCHANTZ, P. M. Epidemiology and pathogenesis of zoonotic toxocariasis. Epidemiologic Reviews, v. 3, p. 230-250, 1981.

9 ZEVALLOS LESCANO, S. A. Estudo epidemiológico da toxocaríase na área urbana de Lima, Peru. 1991.90 f. Dissertação (Mestrado em Parasitologia) - Instituto de Ciências Biomédicas da Universidade de São Paulo, São Paulo, 1991.

10 CHIEFFI, P. P.; MÜLLER, E. E. Prevalência de parasitismo por Toxocara canis em cães e presença de
Visceral Larva Migrans Syndrome.

Zoonosis. ovos de Toxocara sp no solo de localidades públicas da zona urbana do município de Londrina, estado do Paraná, Brasil. Revista de Saúde Pública, v. 10, p. $367-$ 372, 1976.

11 BEZERRA, J. F. Soroprevalência da toxocaríase humana em crianças residentes no município de Moreno-PE. 2002. 57 f. Monografia (Conclusão do Curso de Graduação) - Centro de Ciências Biológicas, Universidade Federal de Pernambuco, Pernambuco, 2002.

12 IBGE. Censo Demográfico 2000. Disponível em: $<$ www.ibge.gov.br/home/estatistica/população/ censo2000 >. Acesso em: 7 mar. 2003.

13 DADA, B. J. A new technique for the recovery of Toxocara eggs of soil. Journal of Helminthology, v. 53, p. 141-144, 1979.

14 BORG, O. A.; WOODRUFF, A. W. Prevalence of infective ova of Toxocara species in public places. British Medical Journal, v. 24, p. 470-472, 1973.

15 COSTA-CRUZ, J. M.; NUNES, R. S.; BUSO, A. B. Presença de ovos de Toxocara spp. em praças públicas da cidade de Uberlândia, Minas Gerais, Brasil. Revista do Instituto de Medicina Tropical de São Paulo, v. 36 n. 1, p. 39-42, 1994.

16 PESSÔA, S. B.; MARTINS, A. V. Parasitologia médica. $11^{a}$ ed. Rio de Janeiro-RJ, Guanabara Koogan: 1988. $872 \mathrm{p}$.

17 SANTARÉM, V. A.; SARTOR, I. F.; BERGAMO, F. M. M. Contaminação, por ovos de Toxocara spp, de parques e praças públicas de Botucatu, São Paulo, Brasil. Revista da Sociedade Brasileira de Medicina Tropical, v. 31, n. 6, p. 529-539, nov. dez. 1998.

18 NUNES, C. M.; SINHORINE, I. L.; OGASSAWARA, S. Influence of soil texture in the recovery of Toxocara canis eggs by a flotation method. Veterinary Parasitology, v. 53, p. 269-274, 1994. 
19 MACIEL, C. C. R. Prevalência das parasitoses intestinais em dois bairros do município de Moreno. 2000. 43 f. Monografia (Trabalho de Conclusão de Curso de Especialização) - Instituto de Ciências Biológicas, Universidade de Pernambuco, Pernambuco, 2000.

20 GOMES, F. A. Prevalência das parasitoses intestinais em áreas rurais do município de Moreno-PE. 2001. 29

f. Monografia (Trabalho de Conclusão de Curso de Especialização) - Instituto de Ciências Biológicas, Universidade de Pernambuco, Pernambuco, 2001.

21 HABLUETZEL, A. et al. An Estimation of Toxocara canis Prevalence in Dogs, Environmental Egg Contamination and Risk of Human Infection in the Marche Region of Italy. Veterinary Parasitology, v. 113, p. 243-252, 2003.

22 SHARGHI, N. et al. Environmental Exposure to Toxocara as a Possible Risk Factor for Asthma: A ClinicBased Case-Control Study. Clinical Infectious Diseases, v. 32 , p. 111-116, 2001 